\title{
Overproduction of cyclo-oxygenase-2 (COX-2) is involved in the resistance to apoptosis in vascular smooth muscle cells from diabetic patients: a link between inflammation and apoptosis
}

\author{
S. Redondo • E. Ruiz • A. Gordillo-Moscoso • \\ J. Navarro-Dorado $•$ M. Ramajo $\cdot$ E. Rodríguez • \\ F. Reguillo $\cdot$ M. Carnero $\cdot$ M. Casado $\cdot$ T. Tejerina
}

Received: 29 June 2010 / Accepted: 27 September 2010 /Published online: 19 October 2010

(C) Springer-Verlag 2010

\begin{abstract}
Aims/hypothesis Inflammation is a common feature in cardiovascular diseases, including diabetes mellitus. In addition to the well-known inflammatory role of cyclooxygenase-2 (COX-2), this protein has also been implicated in apoptosis resistance in tumour cells. Vascular smooth muscle cells (VSMC) from diabetic patients are also resistant to apoptosis because of an increased abundance of B cell lymphoma 2 protein (BCL2). In this work, we investigated whether overproduction of COX-2 was involved in the resistance to apoptosis in VSMC from diabetic patients. Methods VSMC were obtained from internal mammary arteries from patients who had undergone coronary artery bypass graft surgery. Apoptosis was measured by DNA fragmentation, BCL2 degradation and cytochrome $c$ release. Results Apoptosis induced by C-reactive protein in cells from non-diabetic patients was mediated by COX-2. VSMC
\end{abstract}

S. Redondo $(\bowtie) \cdot$ E. Ruiz $\cdot$ A. Gordillo-Moscoso $\cdot$

J. Navarro-Dorado $\cdot$ M. Ramajo $\cdot$ T. Tejerina $(\bowtie)$

Department of Pharmacology, School of Medicine,

Universidad Complutense,

28040 Madrid, Spain

e-mail: santiredondo@hotmail.com

e-mail: teje@med.ucm.es

E. Rodríguez $\cdot$ F. Reguillo $\cdot$ M. Carnero

Service of Cardiac Surgery, Hospital Clínico San Carlos,

Madrid, Spain

\section{Casado}

Institute of Biomedicine of Valencia,

Spanish Council for Scientific Research (IBV-CSIC),

Valencia, Spain from diabetic patients showed higher basal levels of COX-2 compared with those from non-diabetic patients. Transfection of VSMC from non-diabetic patients with a plasmid containing $C O X-2$ (also known as PTGS2) increased basal production of COX-2 and BCL2 and mimicked the resistance to apoptosis that occurs in diabetic patients. We also found a significant correlation $(R=0.846, p=0.016)$ between COX-2 and BCL2 production in arterial rings from diabetic patients measured by confocal microscopy. However, inhibition of COX-2 production by small interfering RNA proved unable to reverse BCL2 production in diabetic VSMC.

Conclusions/interpretation These results suggest a link between inflammation (COX-2) and apoptosis resistance (BCL2) in the arteries of diabetic patients. This relationship is not causative and the common production of these two proteins may be co-regulated by shared regulatory elements in diabetes.

Keywords Apoptosis $\cdot$ COX-2 $\cdot$ Diabetic patients $\cdot$ Human vascular smooth muscle cells $\cdot$ Inflammation

$\begin{array}{ll}\text { Abbreviations } & \\ \text { BCL2 } & \text { B cell lymphoma 2 } \\ \text { COX } & \text { Cyclo-oxygenase } \\ \text { CRP } & \text { C-reactive protein } \\ \text { pcDNA-COX-2 } & \text { Plasmid encoding COX-2 } \\ \text { pcDNA-Gfp } & \begin{array}{l}\text { Plasmid encoding green fluorescent } \\ \text { protein }\end{array} \\ \text { VSMC } & \text { Vascular smooth muscle cells } \\ \text { siRNA } & \text { Small interfering RNA } \\ \text { TBS-T } & \text { TRIS-buffered saline-Tween 20 }\end{array}$




\section{Introduction}

Epidemiological studies show that the prevalence of diabetes mellitus has increased worldwide in the last years $[1,2]$. Inflammation is a common feature in the majority of cardiovascular diseases including atherosclerosis and, especially, the severe atherosclerotic progression that is found in diabetic patients and which has become the major cause of death in this patient group [3]. In this context, several circulating as well as tissue inflammatory markers are increased in patients with diabetes mellitus and atherosclerosis; the enzyme cyclo-oxygenase (COX) is one of these markers.

COX-1 and COX-2, which are the key enzymes in prostaglandin biosynthesis, are thought to be important in maintaining the normal physiological function and certain pathological processes. COX-2, the inducible form of COX, can be produced rapidly under certain circumstances [4]. COX-2 is a rate-limiting enzyme that catalyses the conversion of free arachidonic acid into prostaglandin $\mathrm{H}_{2}$, the first step in the biosynthesis of prostaglandin and thromboxane. A recent paper has shown that COX-2 is highly abundant in vascular smooth muscle cells (VSMC) in a mouse model of type 2 diabetes [5]; additionally, high levels of COX-2 have been found in coronary arterioles obtained from diabetic patients [6]. Beyond the inflammatory role of COX-2, this enzyme has been implicated in the inhibition of apoptotic cell death. Thus, overproduction of COX-2 in tumour cells has been implicated in the resistance to apoptosis $[7,8]$ as it is produced simultaneously with the anti-apoptotic protein B cell lymphoma 2 (BCL2) [9].

Intima-media thickness is a major mechanism of atherosclerotic growth, a process tightly regulated by a delicate balance between vascular cell proliferation and apoptosis [10]. In accordance with this, we have previously shown that VSMC from diabetic patients possess a high proliferative/apoptotic ratio and are resistant to apoptosis induced by $\mathrm{C}$-reactive protein (CRP) via a mechanism involving an increase in BCL2 production [11]. However, the cell pathways that regulate this apoptotic resistance in VSMC from diabetic patients remain unknown.

In the present work we first evaluated the role of COX-2 in CRP-induced apoptosis in VSMC from non-diabetic patients and subsequently we investigated whether overproduction of basal levels of COX-2 might be implicated in the resistance to induced apoptosis in VSMC from diabetic patients.

\section{Methods}

Patients A group of patients was recruited from those undergoing coronary artery bypass graft surgery at the Cardiac Surgery Service (Hospital Clinico San Carlos,
Madrid, Spain). Diabetes mellitus was defined following the criteria established by the ADA [12] as fasting serum glucose concentration $\geq 7 \mathrm{mmol} / 1(126 \mathrm{mg} / \mathrm{dl})$ or use of oral hypoglycaemic drugs or insulin (all our patients had type 2 diabetes). Patient data included: age, sex, active smoker, obesity, total cholesterol, LDL-cholesterol, HDLcholesterol, triacylglycerol, glucose and blood pressure.

Exclusion criteria included: age $>80$ years; pathologies that affect the inflammatory status (for example, renal failure, liver disease) and cancer. Internal mammary arteries were collected by the surgeons during the surgical procedure and used within the next few minutes after the operation. The study was conducted according to the Declaration of Helsinki and we obtained informed consent from all participants before sampling took place.

Cell cultures Human VSMC from internal mammary arteries were cultured from explants in RPMI (Life Technologies, Barcelona, Spain) containing 10\% (vol./vol.) FCS. The cells exhibited typical 'hill and valley' smooth muscle morphology by phase-contrast microscopy and the cultures were stained positively with a monoclonal antismooth $\alpha$-actin antibody. Experiments were performed with VSMC between passages 3 and 5. Biological samples were maintained in a drug-free cell culture medium for at least 2 weeks before a viable cell line was considered to be established.

Measurement of cellular DNA fragmentation VSMC from non-diabetic and diabetic patients were plated on 96 well plates and allowed to attach for $24 \mathrm{~h}$. CRP, $10 \mu \mathrm{g} / \mathrm{ml}$, was added to the culture medium for 12, 24 and $48 \mathrm{~h}$. As some of the in vitro effects of CRP might be due to the sodium azide present in the commercial preparation [13], we also examined the effect of sodium azide $(0.05 \%, 12 \mathrm{~h})$ on apoptosis of human VSMC. To determine the role of COX2 in the apoptosis induced by CRP, we pre-incubated the cells obtained from non-diabetic patients with the COX-2 inhibitor NS-398 $(1 \mu \mathrm{mol} / \mathrm{l})$ for $1 \mathrm{~h}$ prior to CRP treatment. Cellular DNA fragmentation was measured with a commercially available cellular DNA fragmentation ELISA kit (Roche-Boehringer, Barcelona, Spain). Proliferating cells in 96 well microtitre plates were labelled with $10 \mu \mathrm{mol} / 1 \mathrm{BrdU}$ overnight. After treatments, the cells were washed with PBS and incubated with the kit's lysis buffer (BSA, EDTA, and Tween 20) for $30 \mathrm{~min}$ at room temperature, and soluble BrdU-labelled DNA fragments present in the buffer were quantified using the ELISA kit according to the manufacturer's instructions. DNA fragmentation was expressed as fold increase of the control values.

Western blotting To determine the degradation of BCL2 and the production of COX-2 induced by CRP, cells were 
plated onto $60 \mathrm{~mm}$ Petri dishes and allowed to attach for $24 \mathrm{~h}$. Cells were incubated with CRP $10 \mu \mathrm{g} / \mathrm{ml}$ for 4 and $8 \mathrm{~h}$. At the time of harvest, the cells were washed with icecold PBS, lysed on ice with $200 \mu \mathrm{l}$ lysis buffer $(10 \%$ glycerol, $2.3 \%$ SDS, $62.5 \mathrm{mmol} / \mathrm{l}$ TRIS-HCl, pH 6.8 , $150 \mathrm{mmol} / \mathrm{l} \mathrm{NaCl}, 10 \mathrm{mmol} / \mathrm{l}$ EDTA, $1 \mu \mathrm{g} / \mathrm{ml}$ leupeptin, $1 \mu \mathrm{g} / \mathrm{ml}$ pepstatin, $5 \mu \mathrm{g} / \mathrm{ml}$ chymostatin, $1 \mu \mathrm{g} / \mathrm{ml}$ aprotinin, $1 \mathrm{mmol} / \mathrm{l}$ phenylmethylsulphonyl fluoride) and boiled for 5 min. Equal amounts of protein were run on 10\% SDSpolyacrylamide gel electrophoresis. Proteins were then transferred to polyvinylidene difluoride membranes (ImmobilonP; Amersham, Madrid, Spain), and blocked overnight at $4{ }^{\circ} \mathrm{C}$ in blocking solution (3\% BSA in TRIS-buffered salineTween 20 [TBS-T]: $25 \mathrm{mmol} / \mathrm{l}$ TRIS-HCl, $75 \mathrm{mmol} / \mathrm{l} \mathrm{NaCl}$, $\mathrm{pH}=7.4,0.1 \%$ vol./vol. Tween 20). For analysis of BCL2 the blots were incubated for $2 \mathrm{~h}$ with agitation at room temperature in the presence of a specific mouse monoclonal anti-BCL2 (Neomarker, Bionova Científica, Madrid, Spain) at $1.5 \mu \mathrm{g} / \mathrm{ml}$ or in $0.3 \%$ BSA in TBS-T. For analysis of COX2 production, the blots were incubated overnight with agitation at $4^{\circ} \mathrm{C}$ in the presence of a specific mouse monoclonal anti-COX-2 antibody (BD Transduction Laboratories, Madrid, Spain) at 1:1,000 in TBS-T. After washing in TBS-T solution, the blots were further incubated for $1 \mathrm{~h}$ at room temperature with a horseradish-peroxidase-conjugated antimouse secondary antibody diluted 1:10,000 (Santa Cruz Biotechnology, CA, USA) in blocking solution. The blots were then washed five times in TBS-T and antibody-bound protein was visualised using an enhanced chemiluminescence kit (Amersham Biosciences). Smooth muscle $\alpha$-actin was used as a housekeeping protein and it was determined following the same procedure as mentioned above, using a specific anti- $\alpha$-actin mouse monoclonal antibody (SigmaAldrich, Madrid, Spain), at 1:1,000 in TBS-T.

Immunofluorescence staining of cytochrome c Subcellular location of cytochrome $c$ was analysed using confocal images of immunofluorescence-stained samples. VSMC from non-diabetic patients were plated onto coverslips and allowed to attach for $24 \mathrm{~h}$. CRP $(10 \mu \mathrm{g} / \mathrm{ml})$ was added to the cell culture for $8 \mathrm{~h}$. The cells were then treated with Mitotracker (Molecular Probes, Franklin Lakes, IL, USA), $50 \mu \mathrm{mol} / 1$ for $30 \mathrm{~min}$ at $37^{\circ} \mathrm{C}$, to localise mitochondria and were then washed with PBS and fixed with formaldehyde $4 \%$ in PBS for $20 \mathrm{~min}$ at room temperature. Cells were permeabilised with $0.4 \%$ Triton $\mathrm{X}-100$ for $30 \mathrm{~min}$ at room temperature. After blocking with $3 \%$ BSA in PBS, cells were incubated with mouse monoclonal anti-cytochrome $c$ antibody (Neomarkers, Bionova Científica, 1:100) for $1 \mathrm{~h}$. Excess primary antibody was removed by washing with blocking solution, followed by incubation with goat antimouse Alexa 468 (1:100, molecular probes) for $1 \mathrm{~h}$. Cells were then washed four times with blocking buffer every
5 min. Images were captured using a Leica TCS SP2 inverted microscope.

Analysis of COX-2 activity Release of prostaglandin $\mathrm{E}_{2}$ $\left(\mathrm{PGE}_{2}\right)$ into the culture medium was determined as an index of COX-2 activity. VSMC were plated on 24 well plates and allowed to attach for $24 \mathrm{~h}$. Cells were treated with CRP $(10 \mu \mathrm{g} / \mathrm{ml})$ for 6 and $18 \mathrm{~h}$. Cellular medium was collected and $\mathrm{PGE}_{2}$ concentration analysed by enzyme immunoassay (Cayman Chemical, MI, USA) following the manufacturer's instructions. Data were expressed as $\mathrm{pg} \mathrm{PGE}_{2}$ per mg total protein.

Transfection of plasmid coding DNA (pcDNA)-COX-2 Human VSMC were plated onto six-well plates at $70 \%$ confluency and allowed to attach for $24 \mathrm{~h}$. The cells were transfected with a plasmid containing $C O X-2$ (also known as $P T G S 2$ ) (pcDNA-COX-2; construct kindly donated by M. Casado, Instituto de Biomedicina de Valencia, Valencia, Spain). The expression plasmid ( $2 \mu \mathrm{g}$ of pcDNA) encoding human COX-2 protein was transfected into donor cells by a lipid-mediated method using a Fugene6 (Roche-Boehringer) for $3 \mathrm{~h}$. Additionally, transfection of a plasmid encoding green fluorescent protein (pcDNA-Gfp) allowed us to monitor the transfection specificity. Experiments with transfected cells were performed two days after transfection.

Transfection of small interfering RNA Human VSMC from diabetic patients were plated onto cell culture plates and allowed to attach overnight. The cells were then transfected using NeoFX (Ambion, Austin, TX, USA), according to the manufacturer's instructions. Briefly, the cells were detached with trypsin and a mixture of NeoFX diluted in OPTIMEM-serum-free medium (Invitrogen, Carlsbad, CA, USA), and small interfering RNA (siRNA) diluted in OPTI-MEM was prepared. The siRNA was SilencerSelectsiRNA COX-2 for COX-2 inhibition, or control siRNA (Ambion, Austin, TX, USA). The mixture was made at 1:1 and incubated for $5 \mathrm{~min}$ at room temperature. It was added to 6 well cell culture plates, the cells were plated on it and they were allowed to contact the solution by gentle agitation. They were cultured overnight and then OPTI-MEM was changed for RPMI with $10 \%$ FCS. The cells were maintained for $96 \mathrm{~h}$ in order to ensure an appropriate RNA transcription inhibition. Final siRNA concentration was $1 \mathrm{nmol} / 1$ for siRNA-COX-2 and control siRNA.

Materials CRP was purchased from Calbiochem (Bionova Cientifica). NS-398 was purchased from Sigma-Aldrich and the stock solution was dissolved in DMSO. All other reagents were obtained from Sigma unless otherwise stated. Stock solutions were made at $1,000 \times$ concentration. 
Statistical analysis The results are expressed as the mean \pm SD and accompanied by the number of observations. A statistical analysis of the data was carried out by a Student's $t$ test or by a one-way ANOVA when necessary. Differences with a $p$ value of less than 0.05 were considered statistically significant.

\section{Results}

Apoptosis induced by CRP in human VSMC from nondiabetic patients As previously shown by Ruiz et al. [11], CRP induces apoptosis in human VSMC. Figure 1a shows the effect of CRP on DNA fragmentation in human VSMC from non-diabetic patients. CRP at $10 \mu \mathrm{g} / \mathrm{ml}$ significantly increased DNA fragmentation in this cell type, reaching a maximum after $12 \mathrm{~h}$ treatment. Of note, given that we included a control with $0.05 \%$ (wt/vol.) sodium azide, which is frequently present in commercially available CRP [11], we can rule out a non-specific role for this compound (Fig. 1b). Figure 1c, d shows that CRP (10 $\mu \mathrm{g} / \mathrm{ml}, 4$ and $8 \mathrm{~h}$ ) induced degradation of the anti-apoptotic protein BCL2 analysed by western blotting in VSMC from nondiabetic patients. These experimental conditions, however, are unable to trigger apoptosis in VSMC obtained from diabetic patients (Fig. 1e, f). To demonstrate that CRP induced apoptosis by other methods, we also analysed the effect of CRP on two other intermediate pathways in the apoptotic cascade that takes place before DNA fragmentation. As shown in Fig. 1g, CRP was able to induce alterations in the mitochondrial membrane potential in nondiabetic VSMC and, eventually, to release cytochrome $c$ from the mitochondria, as assessed by confocal microscopy. Therefore, CRP induces apoptosis in human VSMC by involving the cytochrome $c$ and BCL2 cascade.

CRP induces COX-2 production in human VSMC from nondiabetic patients We aimed to study whether CRP did indeed induce COX-2 production in these cells and whether this COX-2 was active. COX-2 production was determined by western blotting in cells treated for 4 and $8 \mathrm{~h}$ with CRP $10 \mu \mathrm{g} / \mathrm{ml}$. As shown in Fig. 2a, b, COX-2 production was increased in VSMC from non-diabetic patients as early as $4 \mathrm{~h}$ after stimulation. However, this effect was not found when cells from diabetic patients were analysed (Fig. 2c, d). Further to these results, we analysed the activity of COX-2 measured as the concentration of $\mathrm{PGE}_{2}$ in the supernatant fraction collected from culture of cells, treated with CRP $10 \mu \mathrm{g} / \mathrm{ml}$, from non-diabetic patients. Figure 2e shows that the CRP-induced COX-2 was active as $\mathrm{PGE}_{2}$ levels increased significantly in a time-dependent manner after incubation with CRP. We observed that CRP did not induce apoptosis in cells from diabetic patients (Fig. 2f). To determine the role of $\mathrm{COX}-2$ in the apoptotic effect of CRP on VSMC from non-diabetic patients, cells isolated from non-diabetic individuals were pretreated with the selective COX-2 inhibitor NS-398 (1 $\mu \mathrm{mol} / 1,1 \mathrm{~h})$ before incubation with CRP. Pretreatment with NS-398 significantly reduced the DNA fragmentation induced by CRP in these cells to a threshold similar to that found in cells from diabetic patients (Fig. 2f).

Thus, we aimed to test whether overproduction of basal levels of COX-2 that occur in diabetic patients was also related to apoptosis resistance, as an anti-apoptotic role of COX-2 has been described in tumour cells [7-10].

Transfection of human VSMC from non-diabetic patients with pcDNA-COX-2 To establish a possible role of COX-2 overproduction in the resistance to apoptosis in VSMC, we transfected human VSMC isolated from non-diabetic patients, which initially had low basal levels of COX-2, with a plasmid containing the human gene $C O X-2$ (Fig. 3). Figure $3 \mathrm{a}$ shows the efficacy of cell transfection. Under these experimental conditions, VSMC from diabetic patients had a higher basal level of COX-2, whereas transfected non-diabetic VSMC produced increased levels of COX-2 compared with those transfected with the negative control plasmid (pcDNA-Gfp, Fig. 3b) as it was observed by western blotting. The COX-2 produced was active as observed by the secretion of $\mathrm{PGE}_{2}$ (Fig. 3c). Thus, basal levels of $\mathrm{PGE}_{2}$ were higher in cells transfected with pcDNA-COX-2 compared with control (pcDNA-Gfp). Treatment with CRP also increased $\mathrm{PGE}_{2}$ in cells transfected with this plasmid.

However, CRP and $\mathrm{H}_{2} \mathrm{O}_{2}(10 \mu \mathrm{mol} / \mathrm{l}, 12 \mathrm{~h})$ failed to induce DNA fragmentation in cells transfected with $C O X-2$, as shown in Fig. 3d, e, which demonstrated that overproduction of basal levels of COX-2 was able to prevent the apoptosis induced by CRP, as seen in VSMC from diabetic patients.

Relationship between $C O X-2$ and BCL2 A plausible explanation of the effect of COX-2 in diabetic patients would be that increased basal levels of COX-2 may be associated with increased production of BCL2. Thus, we aimed to study whether there was a link between the resistance to apoptosis and the inflammation observed in diabetic arteries. BCL2 and COX-2 production were analysed in cross-section internal mammary artery rings obtained from patients who had undergone coronary artery bypass grafting. We found a significant correlation between the production of BCL2 and COX-2 in these arteries (Fig. 4a, b). Moreover, to study whether the overproduction of COX-2 in VSMC is able to increase BCL2 abundance, VSMC from nondiabetic patients, initially with low levels of both proteins, were transfected with a plasmid containing $C O X-2$ and 
Fig. 1 Apoptosis induced by CRP in human VSMC from non diabetic patients. a Timeresponse curve of induction of apoptosis by CRP $(10 \mu \mathrm{g} / \mathrm{ml})$ in cells from non-diabetic patients. The maximum effect is reached at $12 \mathrm{~h}$. Black squares, control; black triangles, CRP, $10 \mu \mathrm{g} / \mathrm{ml}$. b Comparison of the apoptotic effect induced by CRP

$(10 \mu \mathrm{g} / \mathrm{ml}, 12 \mathrm{~h})$ and sodium azide at $0.05 \%$. Cellular DNA fragmentation was determined by ELISA in cells from nondiabetic patients. Bar graphs show the mean \pm SD from four patients, hexuplicate samples for each. White bar, control; black bar, with CRP $10 \mu \mathrm{g} / \mathrm{ml}, 12 \mathrm{~h}$; cross-hatched bar, sodium azide, $0.05 \%$. * $p<0.05$. c Timedependent degradation of BCL2 induced by CRP, $10 \mu \mathrm{g} / \mathrm{ml}$, analysed by western blotting in non-diabetic patients. d Quantification of BCL2 degradation in non-diabetic patients. White bar, control; black bar, with CRP $10 \mu \mathrm{g} / \mathrm{ml}, 12 \mathrm{~h}$; cross-hatched bar, sodium azide, $0.05 \%$. e Time-dependent degradation of BCL2 induced by CRP analysed by western blotting in diabetic patients. f Quantification of BCL2 degradation in diabetic patients. Bar graphs show the mean \pm SD from four patients, duplicate samples for each. ${ }^{*} p<0.05$. White bar, control; black bar, with CRP

$10 \mu \mathrm{g} / \mathrm{ml}, 12 \mathrm{~h}$; cross-hatched bar, sodium azide, $0.05 \%$. g Subcellular location of cytochrome $c$ (Cyt $c$, green staining) and mitochondrial damage (red staining) induced by CRP $(10 \mu \mathrm{g} / \mathrm{ml}, 8 \mathrm{~h})$ in VSMC from non-diabetic patients. Scale bars, $40 \mu \mathrm{m}$ a

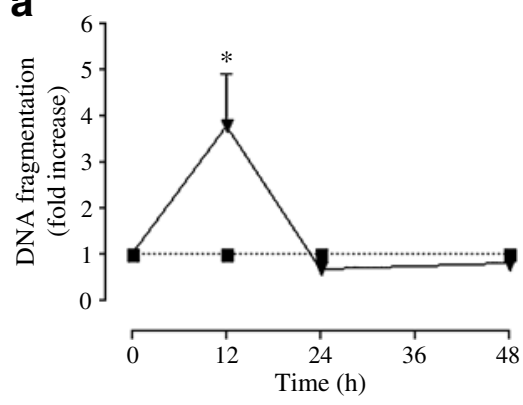

C

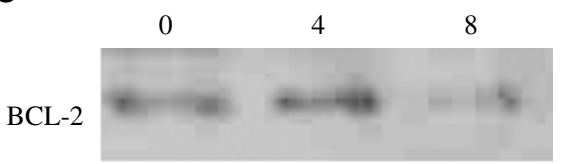

$\alpha$-Actin

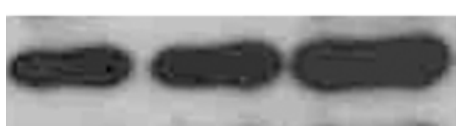

e

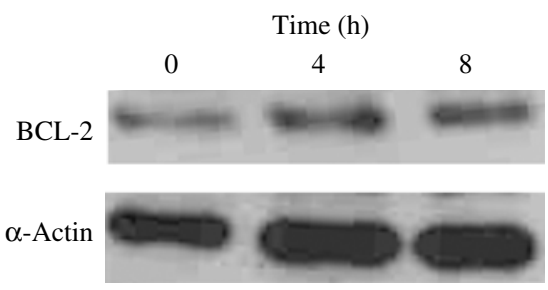

b

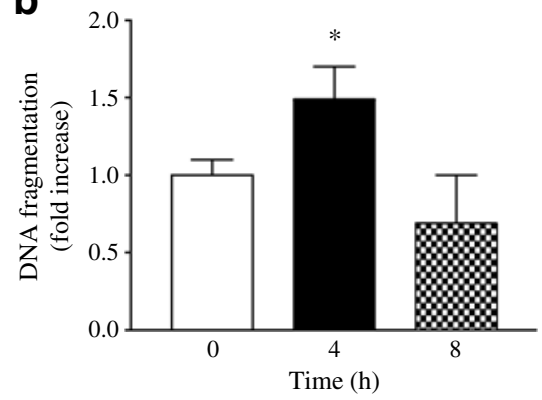

d

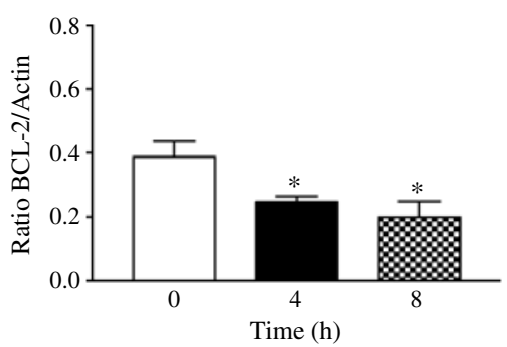

f

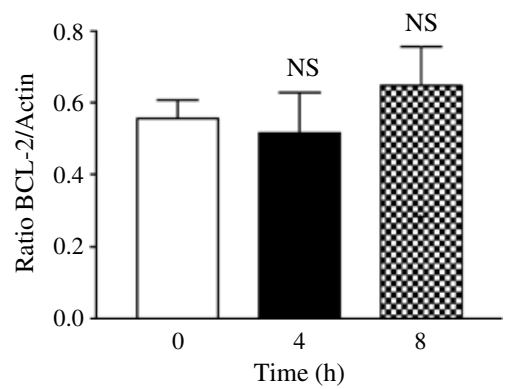

g
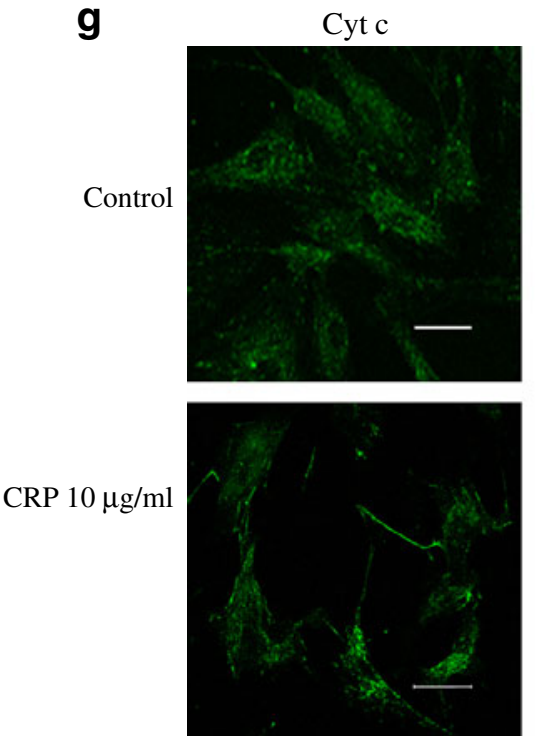
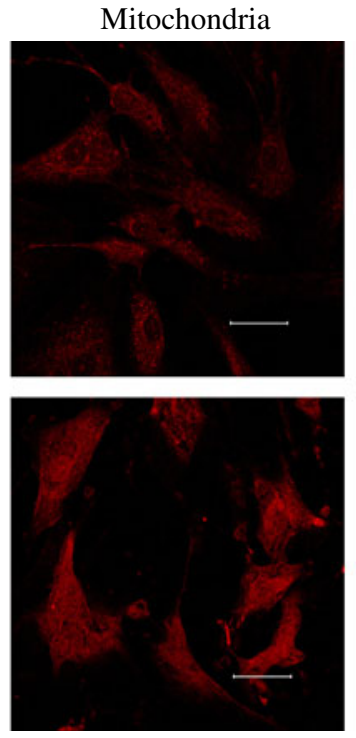
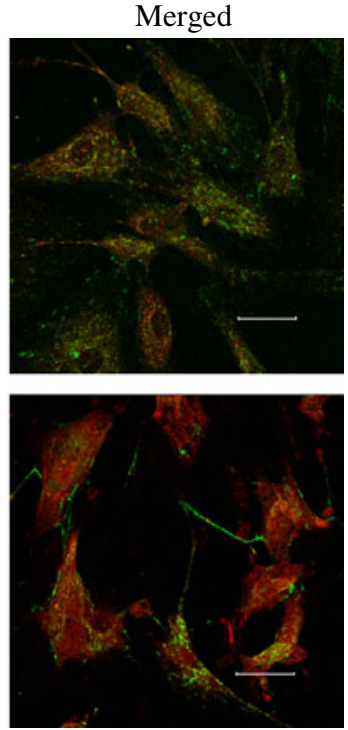

BCL2 content was determined by western blotting. As shown in Fig. 4c, d, levels of BCL2 were higher in VSMC from diabetic patients; however, production of BCL2 was significantly increased in non-diabetic VSMC transfected with pcDNA-COX-2, reaching a similar level to that found in VSMC from diabetic patients.
Effect of transfection of VSMC from diabetic patients with siRNA As shown in Fig. 5a, b, c, when diabetic cells were transfected with siRNA for COX-2, the production of BCL2 did not decrease in a significant manner. This supports the idea that, although COX-2 abundance is associated with BCL2 content, this relationship is not exclusive and other 


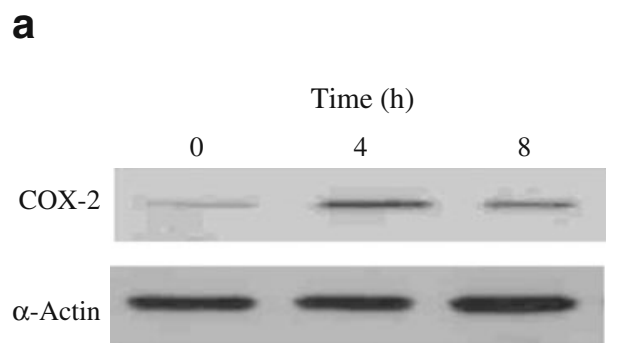

C
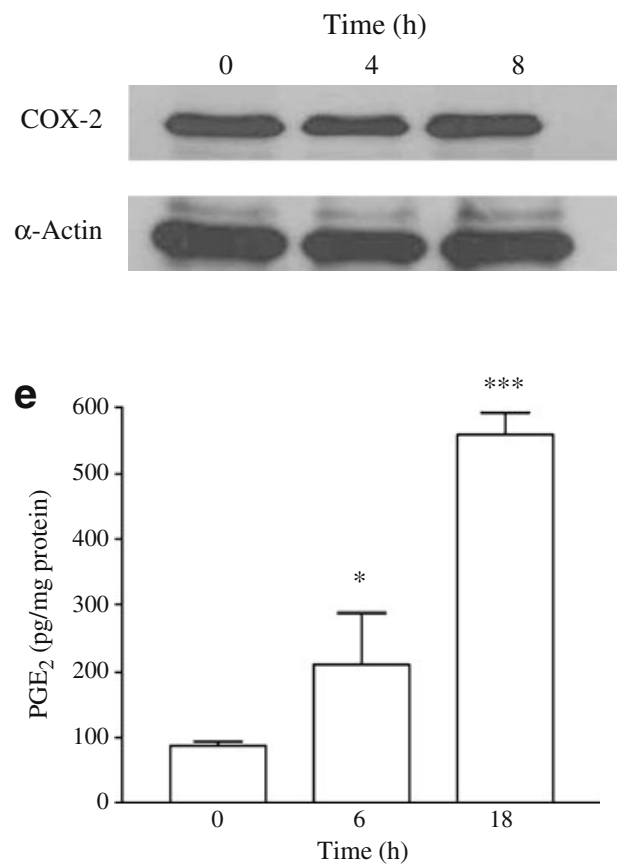

Fig. 2 Production of COX-2 induced by CRP. a Human VSMC from non-diabetic patients were treated with CRP $10 \mu \mathrm{g} / \mathrm{ml}$ for the time indicated and COX-2 production was analysed. b Bar graph shows the densitometric analysis of three independent experiments in cells from non-diabetic participants, each performed in duplicate. c Production of COX-2 was assessed by western blot in VSMC from diabetic patients. d Bar graphs shows the densitometric analysis of three independent experiments in these diabetic cells, each performed in duplicate. e Analysis of COX-2 activity measured as $\mathrm{PGE}_{2}$ production in human VSMC from non-diabetic patients treated with CRP $10 \mu \mathrm{g} / \mathrm{ml}$ for the

additional factors may be implicated in the augmented BCL2 production found in diabetic VSMC.

\section{Discussion}

Our results demonstrate for the first time the role of COX-2 production in the resistance to apoptosis in diabetic human VSMC induced by BCL2 in vitro and ex vivo. Results can be summarised as follows: (1) the inducible enzyme COX-2
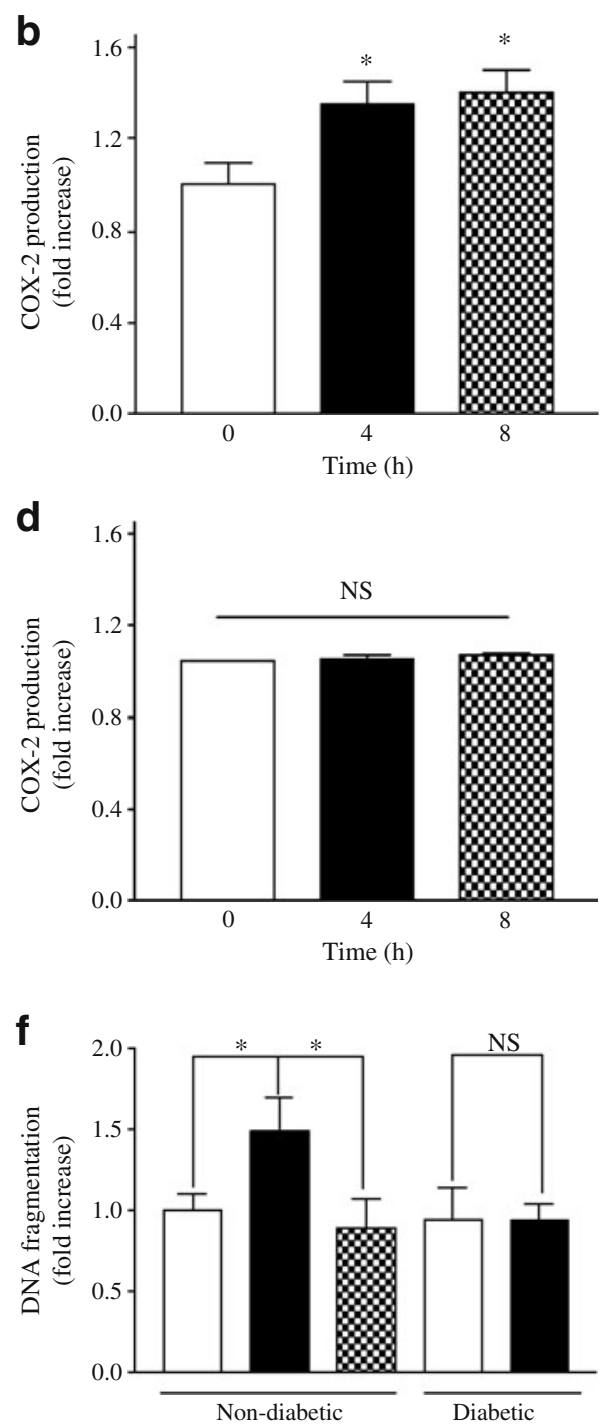

time indicated. The bar graph shows the mean \pm SD for four patients, with quadruplicate samples for each. $\mathbf{f}$ DNA fragmentation in VSMC from diabetic and non-diabetic patients. Human VSMC were pretreated with the selective COX-2 inhibitor NS-398 at $1 \mu \mathrm{mol} / 1$ for $1 \mathrm{~h}$ prior to CRP $(10 \mu \mathrm{g} / \mathrm{ml}, 12 \mathrm{~h})$. Cellular DNA fragmentation was determined by ELISA. The bar graph shows the mean \pm SD for four patients, with hexuplicate samples for each. White bar, control; black bar, with CRP $10 \mu \mathrm{g} / \mathrm{ml}, 12 \mathrm{~h}$; cross-hatched bar, + NS 398, $1 \mu \mathrm{mol} / \mathrm{l}$. $* p<0.05, * * * p<0.001$

played a role in the apoptotic effect of CRP on VSMC from non-diabetic patients; (2) VSMC from diabetic patients were resistant to CRP-induced apoptosis although they showed increased basal levels of COX-2; (3) transfection of the construct pcDNA-COX-2 into human VSMC from nondiabetic patients increased the basal levels of COX-2 and the anti-apoptotic protein BCL2 and made these cells resistant to CRP-induced apoptosis; (4) in agreement with these findings, arteries isolated from diabetic patients showed a good correlation between basal levels of COX-2 
a

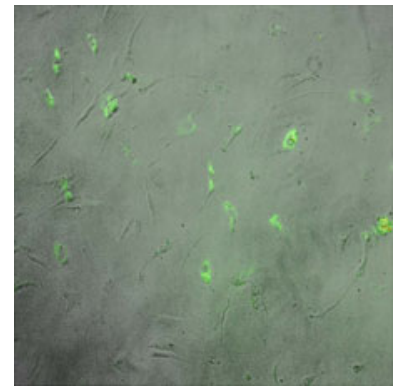

b

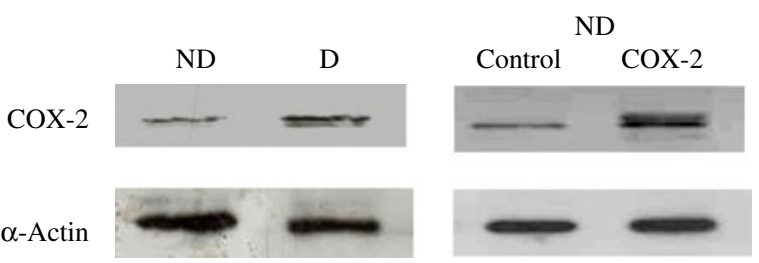

C

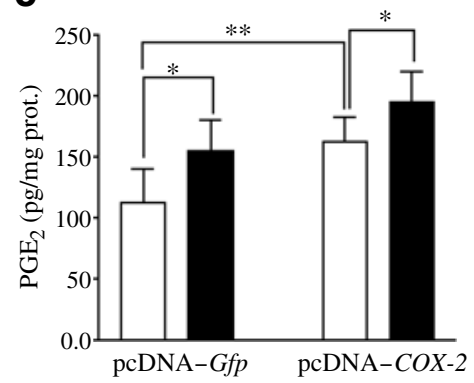

d

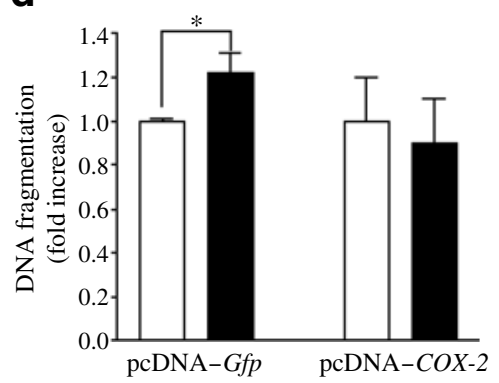

e

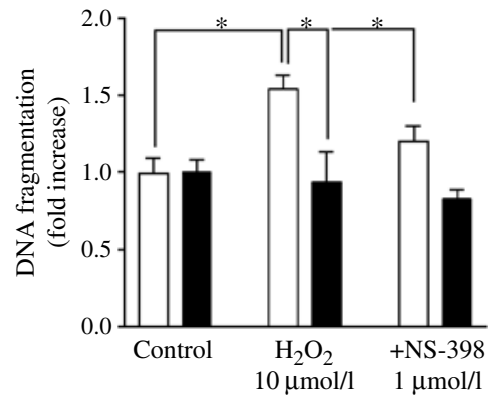

Fig. 3 Overproduction of pcDNA-COX-2 in human VSMC from non-diabetic patients. Human VSMC from non-diabetic patients were transfected with pcDNA encoding green fluorescent protein (GFP) or COX-2. a Microphotograph shows the efficacy of cell transfection. $\mathbf{b}$ Abundance of COX-2 was analysed by western blotting in transfected cells obtained from non-diabetic patients and compared with diabetic ones. c COX-2 activity was measured as $\mathrm{PGE}_{2}$ production in transfected non-diabetic cells treated or untreated with CRP $(10 \mu \mathrm{g} / \mathrm{ml}$, $6 \mathrm{~h}), n=4$. White bar, control; black bar, CRP $10 \mu \mathrm{g} / \mathrm{ml}, 6 \mathrm{~h}$. d DNA fragmentation induced by CRP $(10 \mu \mathrm{g} / \mathrm{ml}, 12 \mathrm{~h})$ in transfected nondiabetic cells. White bar, control; black bar, CRP $10 \mu \mathrm{g} / \mathrm{ml}, 12 \mathrm{~h}$. e DNA fragmentation induced by $\mathrm{H}_{2} \mathrm{O}_{2}(10 \mu \mathrm{mol} / 1,12 \mathrm{~h})$ in transfected non-diabetic cells, and role of the selective COX-2 inhibitor NS-398. Cellular DNA fragmentation was determined by ELISA. White bar, pcDNA-Gfp; black bar, pcDNA-COX-2. Bar graph shows the mean \pm SD from four patients, with hexuplicate samples for each. ${ }^{*} p<0.05$, $* * p<0.01$. ND, non-diabetic; $\mathrm{D}$, diabetic
Fig. 4 Relationship between COX-2 and BCL2. a Correlation of COX-2 and BCL2 levels in the media layer of human internal mammary artery rings from diabetic patients was analysed by immunofluorescence in cross-sectional artery rings. Scale bars, $80 \mu \mathrm{m}$. b Pearson correlation analyses of the relationship between both proteins, $R=0.846, p=0.016$, $n=7$ patients. c Production of BCL2 in human VSMC from non-diabetic patients transfected with pcDNA-COX-2, compared with VSMC obtained from diabetic patients. d Quantification of the ratio BCL $2 / \alpha$-actin. Bar graph shows the mean \pm SD from four patients, with duplicate samples for each. ${ }^{*} p<0.05$. $\mathrm{ND}$, non-diabetic; $\mathrm{D}$, diabetic a

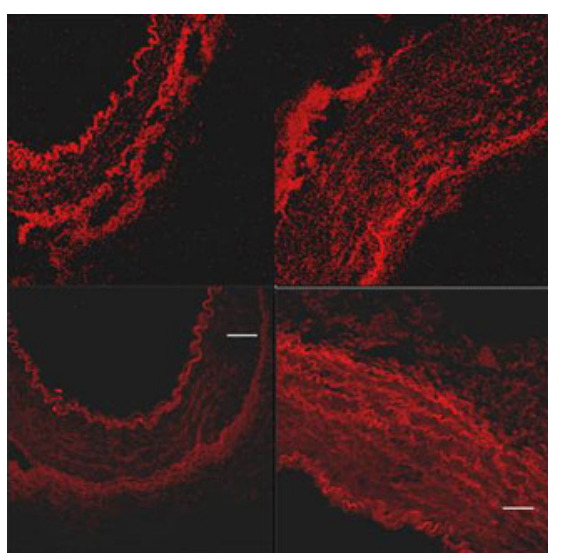

C

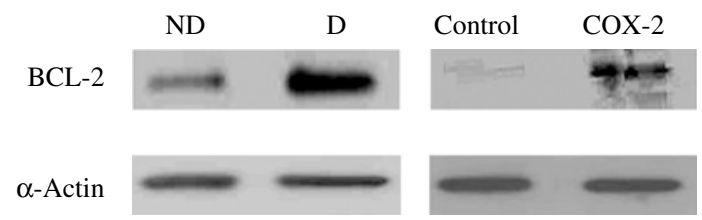

b
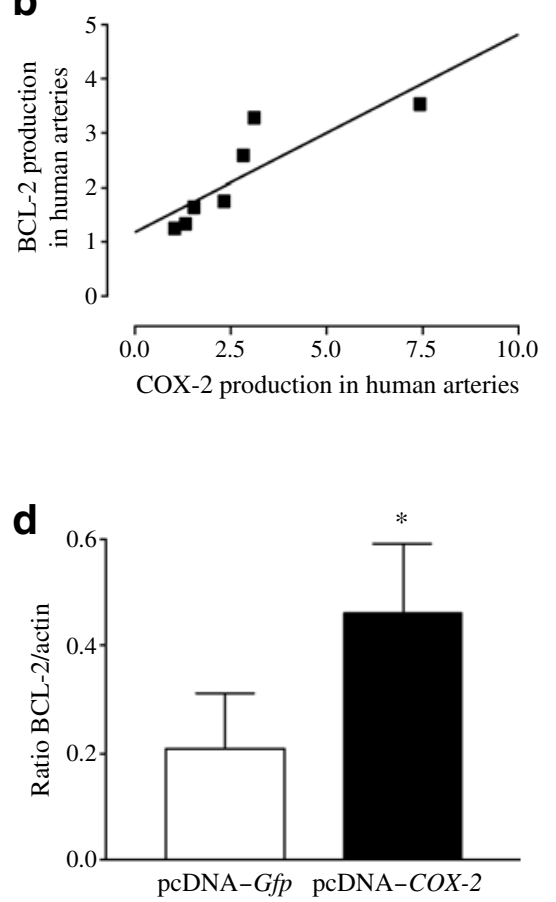
a

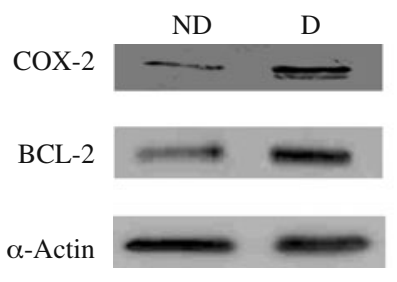

b

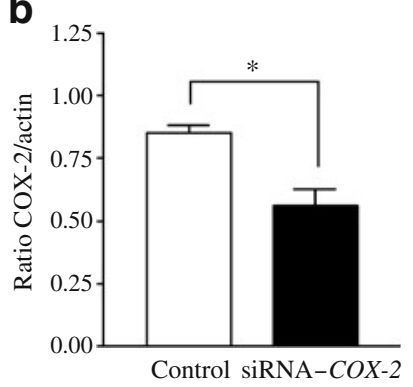

$\mathrm{D}$

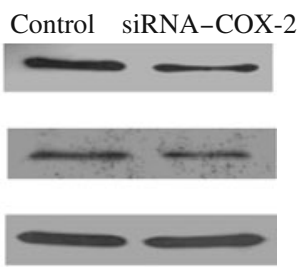

C

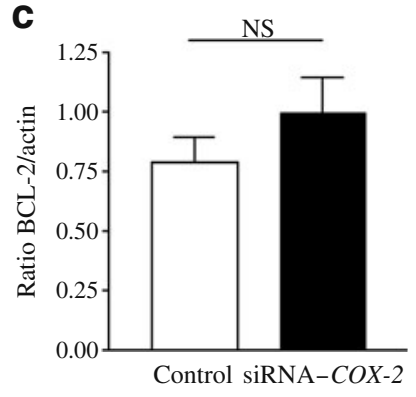

Fig. 5 Role of siRNA. a The effect of siRNA on BCL2 production in human VSMC from diabetic patients, and compared with that found in cells from non-diabetic participants. The cells were transfected with siRNA-COX-2 or control siRNA (each one at $1 \mathrm{nmol} / \mathrm{l}$ ). Western blot experiments were conducted as stated in Methods. The figure shows a representative blot obtained from three different experiments. b Quantification of the above-mentioned set of western blot experiments for COX-2 production, as a measurement of the efficacy of the transfection. c Quantification of BCL2 production. Graphs show the mean $\pm \mathrm{SD}$ of three independent experiments, which were performed with cells from three different diabetic patients. ${ }^{*} p<0.05$. ND, nondiabetic; $\mathrm{D}$, diabetic

and BCL2; but (5) nevertheless, COX-2 is not a causal factor involved in the augmented BCL2 production found in diabetic VSMC, as increased BCL2 production in VSMC isolated from diabetic patients takes place even when COX2 production is repressed by siRNA inhibition. Thus, these two proteins may be co-regulated by common regulatory elements.

We have reported previously that CRP induced apoptosis selectively in VSMC isolated from non-diabetic patients, but not in cells isolated from diabetic patients [11]. The reason for this difference seemed to be the increased basal abundance of the anti-apoptotic protein BCL2 in the VSMC of the media layer in diabetic patients. However, the underlying mechanism to explain this finding remained obscure. The present work, however, highlights that COX-2 is associated with increased BCL2 production and subsequent apoptosis resistance in VSMC obtained from diabetic patients. This association is not a cause-effect relationship, but it may be mediated by common regulatory elements that affect the production of both proteins at the same time.

Inflammatory processes play a key role in the pathogenesis of cardiovascular diseases [14]. Epidemiological studies have shown that increases in serum CRP levels above $3 \mu \mathrm{g} / \mathrm{ml}$ are associated with a high risk of future cardiovascular events in patients with coronary artery disease [15]. CRP has been implicated in many cellular effects; among these, CRP has been shown to induce apoptosis in VSMC isolated from coronary arteries [16] by a mechanism involving the growth arrest and DNA damage inducible protein 153 (GADD153). In the present study, CRP was able to induce apoptosis in VSMC from nondiabetic patients by involving the BCL2 and cytochrome $c$ cascade (Fig. 1).

Subsequently, we analysed whether CRP induced COX2 production and whether this enzyme was active. Figure 2 shows that COX-2 was produced after 4 and $8 \mathrm{~h}$ treatment with CRP. These time-dependency experiments revealed that COX-2 levels reached a maximal value after $4 \mathrm{~h}$ and were maintained for at least $8 \mathrm{~h}$ after treatment. However, COX-2 activity, measured as $\mathrm{PGE}_{2}$ production, increased in a time-dependent fashion from 6 to $18 \mathrm{~h}$. The reason for this apparent disparity might be that CRP could also induce the production of PGE synthase, which would further increase the levels of $\mathrm{PGE}_{2}$ secretion even though the raw levels of COX-2 remained constant. In this context, Jakobsson et al. [17] showed that COX-2 and PGE synthase are co-regulated by inflammatory stimuli and $\mathrm{PGE}_{2}$ biosynthesis and may depend on the presence of both enzymes. In accordance with this, bacterial lipopolysaccharide has been shown to enhance both COX-2 production and $\mathrm{PGE}_{2}$ synthase activity in murine macrophages and also in human monocytes [18, 19].

We found that internal mammary arteries, as well as isolated VSMC, from diabetic patients showed higher basal levels of COX-2 compared with non-diabetic patients (Figs 2, 3 and 5). In a similar manner, VSMC from diabetic patients had a higher abundance of the anti-apoptotic protein BCL2. In accordance with this increase in BCL2 production, these cells were resistant to apoptosis when they were treated with CRP, although the selective COX-2 inhibitor NS-398 blocked the DNA fragmentation induced by CRP in non-diabetic patients (Fig. 2f).

Thus, our findings suggest an anti-apoptotic role of COX-2 production and a pro-apoptotic role of $\mathrm{PGE}_{2}$ secretion. This apparent discrepancy merits some comments. First, our finding of increased apoptosis induced by $\mathrm{PGE}_{2}$ synthesis reproduces what has been found in other human studies that link this mechanism to the pathogenesis of abdominal aortic aneurysms [20]. Second, some crosstalks have been described among COX-2 regulation and production of many other proteins involved in intracellular inflammation and subsequent apoptosis resistance, such as nuclear factor of kappa light polypeptide gene enhancer in $B$ cells 1 (NFkB) [21]. However, due to the close relationship between COX-2 and BCL2 in diabetic vessels (Fig. 4a, b), this co-production of COX-2 and BCL2 and subsequent apoptosis resistance seems to predominate in 
the diabetic state. Of note, our transfection experiments show a lower induction of $\mathrm{PGE}_{2}$ release after COX-2 production compared with that found after CRP treatment (Fig. 3c). This suggests that under high production conditions an incomplete coupling of COX-2 for $\mathrm{PGE}_{2}$ generation takes place. Whether the anti-apoptotic effect of COX-2 overproduction in human VSMC is caused by an uncoupling of $\mathrm{PGE}_{2}$ production towards other nonapoptotic prostanoids remains unknown and will be the goal of future research efforts. However, this mechanism seems plausible, as a glucose-induced increased COX-2 production and a subsequent misbalance towards a higher thromboxane and a lower prostacyclin production has been demonstrated in human endothelial cells [22].

In order to study COX-2 production and apoptosis resistance from a cause-effect point of view, we transfected non-diabetic VSMC with a plasmid encoding COX-2. Under these circumstances, these cells produced high basal levels of COX-2, similar to cells from diabetic patients (Fig. 3b). VSMC transfected with $C O X-2$ behaved similarly to cells from diabetic patients; they were resistant to apoptosis induced by CRP, which agrees with the finding of increased basal levels of the anti-apoptotic protein BCL2 in isolated VSMC as well as in cross-sections of internal mammary arteries from diabetic patients [11]. To determine the possible relationship between COX-2 and BCL2 in isolated arteries from atherosclerotic patients, we analysed the abundance of basal levels of COX-2 and BCL2, measured by confocal microscopy. A significant correlation was found between basal production of COX-2 and BCL2 (Fig. 4a, b).

Accordingly, in vitro experiments showed that VSMC from non-diabetic patients transfected with $C O X-2$ also had higher basal levels of the anti-apoptotic protein BCL2, which demonstrates a link between these two proteins (Fig. 4c, d). Of note, the inverse manipulation did not exert a related effect. When $C O X-2$ transcription was inhibited by siRNA, VSMC from diabetic patients still exhibited a high abundance of the anti-apoptotic protein BCL2 (Fig. 5a, b, c). This reinforces the idea that COX-2 does not play a causative role in BCL2 production. On the contrary, however, production of both proteins seems to be co-regulated by common mediators.

In conclusion, our results show a link between the production of the inflammatory protein $\mathrm{COX}-2$ with the production of BCL2 and apoptosis resistance in VSMC obtained from internal mammary arteries from diabetic patients, which ties in with a significant correlation between COX-2 and BCL2 production in the entire vessels obtained from diabetic patients. However, this mechanism does not seem to exert its effect in an exclusive cause-dependent manner, as siRNA for $C O X-2$ is unable to decrease the production of BCL2 in non-diabetic and diabetic VSMC.
This lack of causative role suggests the existence of common regulatory elements for both genes in the diabetic state. Further investigations will determine the exact mechanistic pathways involved in the link between COX-2 and BCL2 in VSMC.

Acknowledgements This work was funded by Fondo de Investigaciones Sanitarias FISPI080920 (Health Research Fund from the Spanish Ministry of Health), by Red Temática de Investigación Cardiovascular RECAVA RD/06/0014/1007 and RD/06/0014/0025 (Instituto de Salud Carlos III) and by the Spanish Ministry of Science and Innovation (SAF2009-12602). We are grateful to J. A. Paramo (CIMA, Pamplona, Spain) for helping us with the $\mathrm{PGE}_{2}$ analysis and to $\mathrm{F}$. Ortego (Department of Pharmacology, School of Medicine, Universidad Complutense, Madrid, Spain), for his technical assistance.

Duality of interest The authors declare that there is no duality of interest associated with the manuscript.

\section{References}

1. Mokdad AH, Ford ES, Bowman BA et al (2000) Diabetes trends in the US: 1990-1998. Diabetes Care 23:1278-1283

2. Mokdad AH, Bowman BA, Ford ES, Vinicor F, Marks JS, Koplan JP (2001) The continuing epidemics of obesity and diabetes in the United States. JAMA 286:1195-1200

3. Natali A, Vichi S, Landi P, Severi S, L'Abbate A, Ferrannini E (2000) Coronary atherosclerosis in type II diabetes: angiographic findings and clinical outcome. Diabetologia 43:632-641

4. Davidge ST (2001) Prostaglandin H synthase and vascular function. Circ Res 89:650-660

5. Guo Z, Su W, Allen S, Daugherty A, Smart E, Gong MC (2005) COX-2 upregulation and vascular smooth muscle contractile hyperreactivity in spontaneous diabetic $\mathrm{db} / \mathrm{db}$ mice. Cardiovascular Res 67:723-735

6. Szerafin T, Erdei N, Fülöp T et al (2006) Increased cyclooxygenase2 expression and prostaglandin-mediated dilation in coronary arterioles of patients with diabetes mellitus. Circ Res 99:e12-e17

7. Liu XH, Kirschenbaum A, Yao S et al (1999) Upregulation of vascular endothelial growth factor by cobalt chloride simulated hypoxia is mediated by persistent induction of cyclooxygenase-2 in a metastatic human prostate cancer cell line. Clin Exp Metastasis 17:687-694

8. Lim HY, Joo HJ, Choi JH et al (2000) Increased expression of cyclooxygenase-2 protein in human gastric carcinoma. Clin Cancer Res 6:519-525

9. Chen XL, Bao-Shan S, Run-Qin S, Zhang J, Wang YL (2005) Relationship between expression and distribution of cyclooxygenase2 and Bcl-2 in human gastric adenocarcinoma. World J Gastroenterol 11:1228-1231

10. Geng YJ, Libby P (2002) Progression of atheroma: a struggle between death and procreation. Arterioscl Thromb Vasc Biol 22: $1370-1380$

11. Ruiz E, Gordillo-Moscoso A, Padilla E et al (2006) Human vascular smooth muscle cells from diabetic patients are resistant to induced apoptosis due to high Bcl-2 expression. Diabetes 55: $1243-1251$

12. American Diabetes Association (2005) Diagnosis and classification of diabetes mellitus. Diabetes Care 28:S37-S42

13. Taylor KE, Giddings JC, van den Berg C (2005) C-reactive protein-induced in vitro endothelial cell activation is an artefact 
caused by azide and lipopolysaccharide. Arterioscler Thromb Vasc Biol 25:1225-1230

14. Arenillas JF, Alvarez-Sabín J, Molina CA et al (2008) Progression of symptomatic intracranial large artery atherosclerosis is associated with a proinflammatory state and impaired fibrinolysis. Stroke 39:1456-1463

15. Haverkate F, Thompson SG, Pyke SD, Gallimore JR (1997) Production of C-reactive protein and risk of coronary events in stable and unstable angina. European Concerted Action on Thrombosis and Disabilities Angina Pectoris Study Group. Lancet 349:462-466

16. Blaschke F, Bruemmer D, Yin F et al (2004) C-reactive protein induces apoptosis in human coronary vascular smooth muscle cells. Circulation 110:579-587

17. Jakobsson J, Thorén S, Morgenstern R, Samuelsson B (1999) Identification of human prostaglandin E synthase: a microsomal, glutathione-dependent, inducible enzyme, constituting a potential novel drug target. Proc Natl Acad Sci USA 96:7220-7225

18. Matsumoto H, Naraba H, Murakami M et al (1997) Concordant induction of prostaglandin $\mathrm{E}_{2}$ synthase with cyclooxygenase-2 leads to preferred production of prostaglandin $\mathrm{E}_{2}$ over thromboxane and prostaglandin $\mathrm{D}_{2}$ in lipopolysaccharide-stimulated rat peritoneal macrophages. Biochem Biophys Res Commun 230: $110-114$

19. Cipollone F, Prontera C, Pini B et al (2001) Overexpression of functionally coupled cyclooxygenase- 2 and prostaglandin $\mathrm{E}$ synthase in symptomatic atherosclerotic plaques as a basis of prostaglandin E(2)-dependent plaque instability. Circulation 104: 921-927

20. Walton LJ, Franklin IJ, Bayston T et al (1999) Inhibition of prostaglandin E2 synthesis in abdominal aortic aneurysms: implications for smooth muscle cell viability, inflammatory processes, and the expansion of abdominal aortic aneurysms. Circulation 100:48-54

21. Isermann B, Vinnikov IA, Madhusudhan T et al (2007) Activated protein $\mathrm{C}$ protects against diabetic nephropathy by inhibiting endothelial and podocyte apoptosis. Nat Med 13:1349-1358

22. Cosentino F, Eto M, de Paolis P et al (2003) High glucose causes upregulation of cyclooxygenase-2 and alters prostanoid profile in human endothelial cells. Circulation 107:1017-1023 